Supporting info for the paper:

Computational Alanine Scanning and Structural Analysis of the SARS-CoV2 Spike Protein/Angiotensin-Converting Enzyme 2 Complex

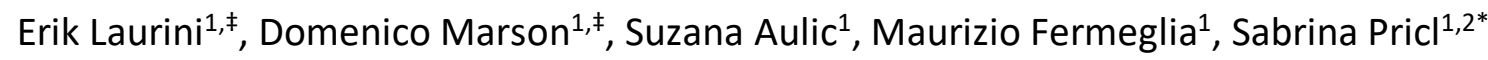

${ }^{1}$ Molecular Biology and Nanotechnology Laboratory (MolBNL@UniTS), DEA, University of Trieste, Trieste, Italy

${ }^{2}$ Department of General Biophysics, Faculty of Biology and Environmental Protection, University of Lodz, Lodz, Poland

Table of Contents

Table S1

Pag. 2

Table S2

Pag. 4

Table S3

Pag. 5

Table S4

Pag. 5

Figure S1

Pag. 6

Extended Methods Section

Pag. 6

References

Pag. 12 
Table S1. Main intermolecular interactions between residues at the protein-protein interface detected during MD simulations of ACE2 in complex with the RBD of SARS-CoV-2 (COV-2). HB = hydrogen bond; $\mathrm{SB}=$ salt bridge; $\mathrm{Cl}=$ contact interactions, including van der Waals/hydrophobic $(v d W / h), \operatorname{polar}(p), \pi / \pi$ and $\pi /$ cation $(\pi / c)$ interactions. In the HB column, s-s indicates side chainside chain interactions while $s-b$ (or $b-s$ ) and $b-b$ indicate side chain-backbone and backbonebackbone interactions, respectively. Intermolecular interactions reported in the original crystal structure $^{1}$ and maintained in the corresponding MD simulation of the viral protein/receptor complex are shown in regular fonts while those newly reported in this work are highlighted in bold.

\begin{tabular}{|c|c|c|c|}
\hline HB & COV-2 & Length ( $\AA$ ) & ACE \\
\hline$S-S$ & Y449 & $2.92 \pm 0.20$ & \multirow{2}{*}{ D38 } \\
\hline s-s & Q498 & $2.92 \pm 0.19$ & \\
\hline$s-s$ & N487 & $3.03 \pm 0.18$ & Q24 \\
\hline$s-s$ & N487 & $2.88 \pm 0.17$ & Y83 \\
\hline s-s & Q493 & $3.04 \pm 0.25$ & K31 \\
\hline$s-s$ & Q493 & $2.94 \pm 0.19$ & E35 \\
\hline$s-s$ & Y505 & $3.15 \pm 0.24$ & E37 \\
\hline$b-b$ & G502 & $2.92 \pm 0.13$ & \multirow{3}{*}{ K353 } \\
\hline$s-s$ & Q498 & $2.87 \pm 0.13$ & \\
\hline$b-s$ & G496 & $2.95 \pm 0.21$ & \\
\hline$S-s$ & T500 & $3.08 \pm 0.23$ & \multirow{2}{*}{ Y41 } \\
\hline$s-s$ & N501 & $3.23 \pm 0.22$ & \\
\hline$S-S$ & Y449 & $3.03 \pm 0.20$ & Q42 \\
\hline $\mathrm{s}-\mathrm{s}$ & T500 & $2.77 \pm 0.16$ & D355 \\
\hline \multirow[t]{3}{*}{ SB } & Cov2 & Length (Å) & \\
\hline & K417 & $3.85 \pm 0.41$ & \\
\hline & R403 & $3.62 \pm 0.39$ & \\
\hline $\mathrm{Cl}$ & COV-2 & ACE & \\
\hline $\mathrm{vdW} / \mathrm{h}$ & G496 & \multirow{2}{*}{ D38 } & \\
\hline $\mathrm{vdW} / \mathrm{h}$ & Y449 & & \\
\hline $\mathrm{vdW} / \mathrm{h}$ & G476 & \multirow{2}{*}{ Q24 } & \\
\hline $\mathrm{vdW} / \mathrm{h}$ & Y489 & & \\
\hline $\mathrm{vdW} / \mathrm{h}$ & F456 & \multirow{3}{*}{$\mathrm{T} 27$} & \\
\hline $\mathrm{vdW} / \mathrm{h}$ & Y489 & & \\
\hline $\mathrm{vdW} / \mathrm{h}$ & Y473 & & \\
\hline$p$ & Y489 & \multirow{2}{*}{ Y83 } & \\
\hline $\mathrm{vdW} / \mathrm{h}$ & F486 & & \\
\hline $\mathrm{vdW} / \mathrm{h}$ & F486 & M82 & \\
\hline $\mathrm{vdW} / \mathrm{h}$ & F486 & L79 & \\
\hline $\mathrm{vdW} / \mathrm{h}$ & F456 & \multirow{2}{*}{ D30 } & \\
\hline $\mathrm{vdW} / \mathrm{h}$ & L455 & & \\
\hline $\mathrm{vdW} / \mathrm{h}$ & Y489 & \multirow{3}{*}{ K31 } & \\
\hline $\mathrm{vdW} / \mathrm{h}$ & L455 & & \\
\hline $\mathrm{vdW} / \mathrm{h}$ & F456 & & \\
\hline$p$ & Y453 & H34 & \\
\hline
\end{tabular}




\begin{tabular}{|c|c|c|}
\hline $\mathrm{vdW} / \mathrm{h}$ & L455 & \\
\hline $\mathrm{p}$ & Y505 & R393 \\
\hline$p$ & T500 & R357 \\
\hline $\mathrm{p}$ & N501 & \\
\hline $\mathrm{vdW} / \mathrm{h}$ & Y505 & K353 \\
\hline $\mathrm{vdW} / \mathrm{h}$ & Y41 & \\
\hline $\mathrm{vdW} / \mathrm{h}$ & Q498 & Q42 \\
\hline $\mathrm{vdW} / \mathrm{h}$ & Q498 & Y41 \\
\hline $\mathrm{vdW} / \mathrm{h}$ & T500 & N330 \\
\hline
\end{tabular}


Table S2. Main intramolecular interactions between residues at the protein-protein interface detected during MD simulations of ACE2 in complex with the RBD of SARS-CoV-2 (COV-2). HB = hydrogen bond; $\mathrm{SB}=$ salt bridge; $\mathrm{Cl}=$ contact interactions, including van der Waals/hydrophobic $(v d W / h)$, polar $(p), \pi / \pi$ and $\pi /$ cation $(\pi / c)$ interactions. In the HB column, s-s indicates side chainside chain interactions while $s-b$ (or $b-s$ ) and $b-b$ indicate side chain-backbone and backbonebackbone interactions, respectively.

\begin{tabular}{|c|c|c|c|c|c|c|c|}
\hline HB & ACE & Length (Å) & ACE & COV-2 & Length ( $\AA$ ) & COV-2 & HB \\
\hline$s-b$ & Q42 & $3.04 \pm 0.18$ & D38 & Y449 & $3.04 \pm 0.18$ & \multirow{2}{*}{ Q498 } & $\mathrm{s}-\mathrm{s}$ \\
\hline$b-s$ & E23 & $3.05 \pm 0.16$ & $\mathrm{~T} 27$ & N501 & $3.02 \pm 0.18$ & & $s-s$ \\
\hline $\mathrm{s}-\mathrm{s}$ & H34 & $3.31 \pm 0.18$ & D30 & Q493 & $3.24 \pm 0.21$ & S494 & $\mathrm{s}-\mathrm{s}$ \\
\hline$s-s$ & D355 & $2.78 \pm 0.15$ & $\mathrm{Y41}$ & & & & \\
\hline \multirow[t]{5}{*}{ SB } & ACE & Length $(\AA ̊)$ & ACE & COV-2 & Length $(\AA ̊)$ & cov-2 & SB \\
\hline & K353 & $3.66 \pm 0.39$ & D38 & R403 & $3.95 \pm 0.37$ & D405 & \multirow[b]{5}{*}{ C } \\
\hline & E35 & $3.94 \pm 0.42$ & K31 & & \multirow[b]{4}{*}{ COV-2 } & & \\
\hline & R393 & $3.93 \pm 0.38$ & E37 & & & & \\
\hline & R357 & $3.68 \pm 0.19$ & D355 & & & & \\
\hline $\mathrm{Cl}$ & ACE & ACE & & & & cov-2 & \\
\hline $\mathrm{vdW} / \mathrm{h}$ & L29 & \multirow{5}{*}{ F28 } & & & \multirow{2}{*}{ K417 } & F456 & $\pi / c$ \\
\hline $\mathrm{vdW} / \mathrm{h}$ & Q76 & & & & & L455 & $\mathrm{vdW} / \mathrm{h}$ \\
\hline $\mathrm{vdW} / \mathrm{h}$ & L79 & & & & \multirow{2}{*}{ R403 } & Y505 & $\pi / c$ \\
\hline $\mathrm{vdW} / \mathrm{h}$ & Y83 & & & & & Y495 & $\mathrm{vdW} / \mathrm{h}$ \\
\hline $\mathrm{vdW} / \mathrm{h}$ & L97 & & & & Y453 & Q493 & $\mathrm{vdW} / \mathrm{h}$ \\
\hline $\mathrm{vdW} / \mathrm{h}$ & Y83 & Q24 & & & F456 & Y473 & $\mathrm{vdW} / \mathrm{h}$ \\
\hline $\mathrm{vdW} / \mathrm{h}$ & L79 & M82 & & & & & \\
\hline$p$ & N330 & & & & & & \\
\hline $\mathrm{p}$ & W48 & R357 & & & & & \\
\hline $\mathrm{vdW} / \mathrm{h}$ & L351 & & & & & & \\
\hline $\mathrm{vdW} / \mathrm{h}$ & L45 & YA1 & & & & & \\
\hline $\mathrm{vdW} / \mathrm{h}$ & L351 & Y4I & & & & & \\
\hline
\end{tabular}


Table S3. Relative binding free energy and its components calculated by the combined computational alanine scanning mutagenesis - interaction entropy approach for the ACE2 residues effectively involved in the binding interface with the S-RBD of SARS-CoV-2 (see the SI Materials and Methods section for details). IE $=$ interaction entropy. $\Delta \Delta G=\Delta G_{W T}-\Delta G_{A L A}$ (see text for details).

\begin{tabular}{|c|c|c|c|c|c|c|c|c|c|c|}
\hline & D38A & Q24A & T27A & F28A & Y83A & M82A & L79A & D30A & K31A & E35A \\
\hline$\Delta \Delta \mathrm{E}_{\mathrm{vdW}}$ & -0.77 & -1.96 & -1.80 & -1.02 & -2.22 & -0.72 & -1.02 & -0.92 & -2.9 & -1.27 \\
\hline$\Delta \Delta \mathrm{E}_{\mathrm{ELE}}$ & -10.26 & -0.99 & -0.30 & -0.09 & -1.85 & 0.01 & -0.03 & -11.36 & 1.35 & -6.31 \\
\hline$\Delta \Delta \mathrm{G}_{\mathrm{SOL}}$ & 7.18 & 0.75 & 0.14 & 0.16 & 1.07 & -0.04 & 0.01 & 9.50 & -3.18 & 5.37 \\
\hline$\Delta \Delta \mathrm{H}$ & -3.85 & -2.20 & -1.96 & -0.95 & -3.00 & -0.75 & -1.04 & -2.78 & -4.73 & -2.21 \\
\hline$\Delta \Delta \mathrm{IE}$ & -1.26 & -0.41 & -0.27 & -0.03 & -0.23 & -0.01 & 0.00 & -1.11 & -0.12 & -0.68 \\
\hline \multirow[t]{2}{*}{$\Delta \Delta \mathbf{G}_{\mathrm{ACE} 2}$} & $\begin{array}{l}-5.11 \\
(0.38)\end{array}$ & $\begin{array}{c}-2.61 \\
(0.37)\end{array}$ & $\begin{array}{c}-2.23 \\
(0.39)\end{array}$ & $\begin{array}{c}-0.98 \\
(0.28)\end{array}$ & $\begin{array}{c}-3.23 \\
(0.40)\end{array}$ & $\begin{array}{c}-0.76 \\
(0.32)\end{array}$ & $\begin{array}{c}-1.04 \\
(0.36)\end{array}$ & $\begin{array}{l}-3.89 \\
(0.32)\end{array}$ & $\begin{array}{l}-4.85 \\
(0.34)\end{array}$ & $\begin{array}{c}-2.89 \\
(0.35)\end{array}$ \\
\hline & H34A & R393A & R357A & E37A & K353A & Q42A & Y41A & D355A & N330A & \\
\hline$\Delta \Delta \mathrm{E}_{\mathrm{vdW}}$ & -1.36 & -0.23 & -1.29 & -1.83 & -2.22 & -0.96 & -2.43 & -0.31 & -0.54 & \\
\hline$\Delta \Delta \mathrm{E}_{\mathrm{ELE}}$ & 4.37 & 6.67 & 5.82 & -11.18 & 5.1 & -1.25 & -3.07 & -7.89 & -0.19 & \\
\hline$\Delta \Delta \mathrm{G}_{\mathrm{soL}}$ & -4.24 & -8.31 & -7.23 & 8.98 & -8.71 & 0.33 & 1.45 & 6.01 & 0.16 & \\
\hline$\Delta \Delta \mathrm{H}$ & -1.23 & -1.87 & -2.70 & -4.03 & -5.83 & -1.88 & -4.05 & -2.19 & -0.57 & \\
\hline$\Delta \Delta \mathrm{IE}$ & -0.54 & -0.46 & -0.62 & -1.09 & -1.36 & -0.31 & -0.38 & -0.99 & -0.11 & \\
\hline$\Delta \Delta \mathbf{G}_{\mathrm{ACE} 2}$ & $\begin{array}{c}-1.77 \\
(0.29) \\
\end{array}$ & $\begin{array}{l}-2.33 \\
(0.39)\end{array}$ & $\begin{array}{c}-3.32 \\
(0.45) \\
\end{array}$ & $\begin{array}{c}-5.12 \\
(0.42)\end{array}$ & $\begin{array}{l}-7.19 \\
(0.54) \\
\end{array}$ & $\begin{array}{c}-2.19 \\
(0.31) \\
\end{array}$ & $\begin{array}{c}-4.43 \\
(0.43) \\
\end{array}$ & $\begin{array}{c}-3.18 \\
(0.41)\end{array}$ & $\begin{array}{c}-0.68 \\
(0.25)\end{array}$ & \\
\hline
\end{tabular}

Table S4. Relative binding free energy and its components calculated by the combined computational alanine scanning mutagenesis - interaction entropy approach for the S-RBD of SARSCoV-2 residues effectively involved in the binding interface with ACE2 (see the SI Materials and Methods section for details). IE = interaction entropy. $\Delta \Delta G=\Delta G_{W T}-\Delta G_{A L A}$ (see text for details).

\begin{tabular}{|c|c|c|c|c|c|c|c|c|c|c|}
\hline & K417A & Y449A & Y453A & L455A & F456A & F486A & N487A & Y489A & Q493A & Q498A \\
\hline$\Delta \Delta \mathrm{E}_{\mathrm{vdW}}$ & -0.53 & -1.45 & -0.14 & -1.34 & -2.08 & -1.59 & -1.32 & -2.19 & -2.19 & -2.95 \\
\hline$\Delta \Delta \mathrm{E}_{\mathrm{ELE}}$ & -12.64 & -2.43 & -0.80 & -0.06 & -0.12 & -0.19 & -1.84 & -1.2 & -2.44 & -3.37 \\
\hline$\Delta \Delta \mathrm{G}_{\mathrm{SOL}}$ & 11.48 & 0.99 & 0.34 & 0.23 & 0.22 & -0.31 & 1.06 & 0.94 & 1.82 & 1.23 \\
\hline$\Delta \Delta \mathrm{H}$ & -1.69 & -2.89 & -0.60 & -1.17 & -1.98 & -2.09 & -2.1 & -2.45 & -2.81 & -5.09 \\
\hline$\Delta \Delta \mathrm{IE}$ & -1.03 & -0.32 & -0.19 & -0.04 & -0.01 & -0.04 & -0.15 & -0.51 & -0.34 & -0.27 \\
\hline \multirow[t]{3}{*}{$\Delta \Delta \mathrm{G}_{\mathrm{CoV}-2}$} & -2.72 & -3.21 & -0.79 & -1.21 & -1.99 & -2.13 & -2.25 & -2.96 & -3.15 & -5.36 \\
\hline & $(0.34)$ & $(0.31)$ & $(0.30)$ & $(0.32)$ & $(0.28)$ & $(0.32)$ & $(0.35)$ & $(0.33)$ & $(0.29)$ & $(0.37)$ \\
\hline & T500A & N501A & Y505A & R403A & & & & & & \\
\hline$\Delta \Delta \mathrm{EvdW}$ & -2.65 & -2.01 & -2.06 & -1.69 & & & & & & \\
\hline$\Delta \Delta \mathrm{E}_{\mathrm{ELE}}$ & -2.26 & -2.6 & -2.09 & 6.27 & & & & & & \\
\hline$\Delta \Delta \mathrm{G}_{\mathrm{SOL}}$ & 0.99 & 2.39 & 1.29 & -7.68 & & & & & & \\
\hline$\Delta \Delta \mathrm{H}$ & -3.92 & -2.22 & -2.86 & -3.10 & & & & & & \\
\hline$\Delta \Delta \mathrm{IE}$ & -0.25 & -0.18 & -0.41 & -1.15 & & & & & & \\
\hline \multirow[t]{2}{*}{$\Delta \Delta G_{\text {CoV-2 }}$} & -4.17 & -2.40 & -3.27 & -4.25 & & & & & & \\
\hline & $(0.36)$ & $(0.28)$ & $(0.31)$ & $(0.39)$ & & & & & & \\
\hline
\end{tabular}




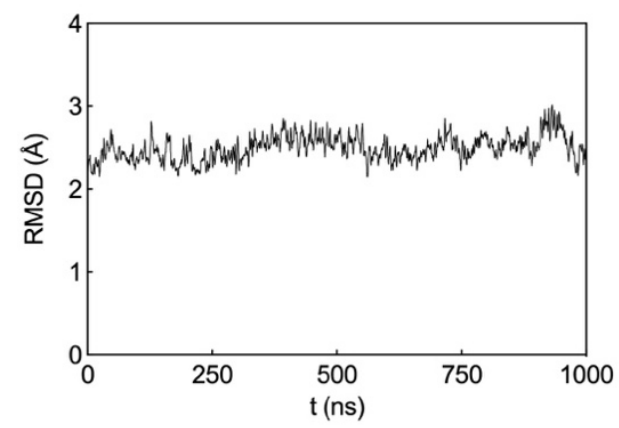

Figure S1. Root-mean-square deviation (RMSD) of the ACE2/S-RBD cov-2 protein complex backbone atoms as a function of MD simulation time.

\section{Extended Methods Section}

The starting structure for the wild type ACE2 protein in complex with the SARS-CoV-2 S-protein receptor binding domain (S-RBD $\left.\mathrm{Cov}_{-2}\right)$ (PDB ID 6MOJ) ${ }^{1}$ was obtained from the RCSB Protein Data Bank. ${ }^{2}$ All residues were protonated at their physiological state by the $\mathrm{H}++$ server (http://biophysics.cs.vt.edu/H++). ${ }^{3}$ The force field parameters for the Zinc atom and its protein bounded residues were obtained with the MCPB.py tools ${ }^{4}$ provided within the AMBER195 suite of programs and GAMESS-US ${ }^{6}$ software using the B3LYP/6-31G* level of theory.

The tleap software provided within AMBER19 was used to assign the ff14SB ${ }^{7}$ and GLYCAM06j- ${ }^{8}$ forcefields to the starting protein/protein structure. The latter was next solvated in a box of TIP3PB ${ }^{9}$ water molecules spanning at least $1.2 \mathrm{~nm}$ from each solute atoms. An appropriate number of $\mathrm{Na}^{+}$ and $\mathrm{Cl}^{-}$atoms were added to neutralize the system and mimic a physiological salt concentration (0.15 M).

While applying a weak restraint $(10 \mathrm{kcal} / \mathrm{mol})$ on the protein's backbone atoms, the simulation box was firstly energy minimized (3000 steps of steepest descent followed by 3000 steps of conjugated gradient algorithms), than heated to $150 \mathrm{~K}$ in $10 \mathrm{ps}$ of canonical ensemble (NVT) molecular dynamics (MD), followed to another 50 ps MD simulation in the isothermal/isobaric ensemble (NPT, $P=1$ atm, maintained by the Berendsen barostat $\left.{ }^{10}\right)$ to reach the target temperature of $300 \mathrm{~K}$. The restraints were then gradually removed in 5 steps $(-2 \mathrm{kcal} / \mathrm{mol}$ per step) of energy minimization (2000 steps of steepest descent followed by 2000 steps of conjugated gradient algorithms). The MD simulation was next carried out without restraints for further $10 \mathrm{~ns}$ in NPT conditions (phase 1); after this time interval, the MD data production run was further continued up to $1 \mu \mathrm{s}$, during which pressure was maintained using the Monte Carlo barostat implemented in AMBER (phase 2). Along the entire MD trajectory, electrostatic interactions were computed by means of the particle mesh Ewald (PME) ${ }^{11}$ algorithm temperature was regulated by the Langevin method ${ }^{12}$ (collision frequency of $3 \mathrm{ps}^{-1}$ ). The SHAKE algorithm ${ }^{13}$ was applied to allow a 2 fs integration time step. All calculations were run with the pmemd module of AMBER19 running on the supercomputer Marconi100 (CINECA, Bologna, Italy) and on our CPU/GPU hybrid cluster. All images were produced by the UCSF Chimera software ${ }^{14}$ and on Prism 8 GraphPad Prism version 8.0.0 for Mac (GraphPad Software, San Diego, California USA, www.graphpad.com).

After the first $5 \mathrm{~ns}$ of the phase $2 \mathrm{MD}$ trajectory, $5 \mathrm{~ns} \mathrm{MD}$ data were selected to calculate enthalpy and entropy contributions. Configurational sampling was preformed accordingly, with a time step of $10 \mathrm{fs}$; thus, a total of 500000 snapshots, sufficient for the interaction entropy (IE) calculations, ${ }^{15-}$ 
${ }^{17}$ were extracted from the relevant MD trajectory for the calculation of the protein/protein residuespecific interactions.

The free energy was calculated for each molecular species (protein/protein complex, ACE2, and S$\mathrm{RBD}_{\text {(ov-2) }}$ in the framework of the MM/PBSA ansatz, ${ }^{18}$ and the protein/protein binding free energy was computed as the difference:

$\Delta \mathrm{G}=\mathrm{G}_{\mathrm{ACE} 2 / \mathrm{S}-\mathrm{RBDCOV}-2}-\left(\mathrm{G}_{\mathrm{ACE2}}+\mathrm{G}_{\mathrm{S}-\mathrm{RBDCOV}-2}\right)=\Delta \mathrm{E}_{\mathrm{vdW}}+\Delta \mathrm{E}_{\mathrm{ELE}}+\Delta \mathrm{G}_{\mathrm{SOL}}-\mathrm{T} \Delta \mathrm{S}=\Delta \mathrm{H}-\mathrm{T} \Delta \mathrm{S}$

in which $\Delta \mathrm{E}_{\mathrm{vdW}}$ and $\Delta \mathrm{E}_{\mathrm{ELE}}$ represent van der Waals and electrostatic molecular mechanics energies, and $\Delta G_{\text {sol }}$ includes the solvation free energy. The internal dielectric constant was set to the values of 2, 3 and 9 for nonpolar, polar, and charged residues, ${ }^{15,19}$ respectively. Lastly, the entropic contribution ( $T \Delta S$ ) was explicitly computed from the MD simulation by using the Interaction Entropy (IE) method. ${ }^{15-17}$ According to this approach, the entropic contribution to $\Delta G$ is determined from fluctuation of the interactions along the MD simulation, and IE is defined as:

$-T \Delta S=K T \ln \left(e^{\beta \Delta E^{I N T}}\right)$

The calculation of IE by equation (2) involves the natural log of an ensemble average of $e^{\beta \Delta E^{I N T}}$, which can be extracted without extra computational cost by numerical integration along the MD trajectories, as follows:

$\left\langle e^{\beta \Delta E^{I N T}}\right\rangle=\frac{1}{N} \sum_{i=1}^{N} e^{\beta \Delta E^{I N T}}\left(t_{i}\right)$

in which $\Delta E^{I N T}=E^{I N T}-\left\langle E^{I N T}\right\rangle$ and the average interaction energy is obtained by:

$\left\langle E^{I N T}\right\rangle=\frac{1}{T} \int_{0}^{T} E^{I N T}(t) d t=\frac{1}{N} \sum_{i=1}^{N} E^{I N T}\left(t_{i}\right)$

The role of the protein/protein interface key residues was studied by performing computational alanine scanning (CAS) experiments. ${ }^{20}$ Accordingly, the absolute binding free energy of each mutant receptor - in which each key residue was replaced by alanine by truncating the mutated residue at the $C \gamma$ atom, and replacing it with a hydrogen - was calculated with the MM/PBSA method. Accordingly, the difference in the binding free energy between the wild-type (WT) protein and its alanine mutant (ALA) counterpart, $\Delta \Delta G$, is given by:

$\Delta \Delta G=\Delta G_{\text {WILD-TYPE }}-\Delta \mathrm{G}_{\mathrm{ALA}}$

Thus, the CAS methodology allows for the estimation of the contribution of a given residue with respect to the overall protein-protein binding free energy; indeed, according to equation (5), a negative value of $\Delta \Delta G$ indicated a favorable contribution for the wild type residue in that position and vice versa.

At the structural level, the stability of the main protein/protein interface intermolecular and intramolecular interactions detected during the MD simulation time interval adopted for the energetic analysis was assessed along the entire duration of the MD run.

\section{Force field parameters for the ACE2 $\mathrm{Zn}^{2+}$ binding site}

Coordinate file for glutamic acid residue in $\mathrm{Zn}^{2+}$ binding site (mol2)

¿ < TRIPOS>MOLECULE

GU1 
SMALL

RESP Charge

\section{a $<$ TRIPOS $>$ ATOM}

$\begin{array}{llllll}1 & \mathrm{~N} & -21.7350 & 17.0320 & -18.4210 & \mathrm{~N} \\ 2 & \mathrm{H} & -22.1320 & 17.8590 & -18.8430 & \mathrm{H} \\ 3 & \mathrm{CA} & -21.5150 & 15.8580 & -19.2530 & \mathrm{CX} \\ 4 & \mathrm{HA} & -21.9560 & 14.9850 & -18.7690 & \mathrm{H} 1 \\ 5 & \mathrm{CB} & -22.2040 & 16.0520 & -20.5960 & 2 \mathrm{C} \\ 6 & \mathrm{HB} 2 & -22.0190 & 17.0550 & -20.9850 & \mathrm{HC} \\ 7 \mathrm{HB} 3 & -21.7690 & 15.3080 & -21.2500 & \mathrm{HC} \\ 8 & \mathrm{CG} & -23.6730 & 15.7410 & -20.6070 & 2 \mathrm{C} \\ 9 & \mathrm{HG} 2 & -24.1890 & 16.4290 & -19.9340 & \mathrm{HC} \\ 10 & \mathrm{HG} 3 & -24.0600 & 15.9040 & -21.6140 & \mathrm{HC} \\ 11 & \mathrm{CD} & -23.9520 & 14.3120 & -20.2020 & \mathrm{CO} \\ 12 & \text { OE1 } & -24.6420 & 14.1190 & -19.1780 & \mathrm{Y} 3 \\ 13 & \text { OE2 } & -23.4730 & 13.3850 & -20.9030 & \mathrm{O} 2 \\ 14 & \mathrm{C} & -20.0270 & 15.5730 & -19.4390 & \mathrm{C} \\ 15 & \mathrm{O} & -19.6160 & 14.4090 & -19.4620 & \mathrm{O}\end{array}$

$\begin{array}{ll}1 & \mathrm{GU} 1 \\ 1 & \mathrm{GU1} \\ 1 & \mathrm{GU1} \\ 1 & \mathrm{GU1} \\ 1 & \mathrm{GU1} \\ 1 & \mathrm{GU1} \\ 1 & \mathrm{GU1} \\ 1 & \mathrm{GU1} \\ 1 & \mathrm{GU1} \\ 1 & \mathrm{GU1} \\ 1 & \mathrm{GU1} \\ 1 & \mathrm{GU1} \\ 1 & \mathrm{GU1} \\ 1 & \mathrm{GU1} \\ 1 & \mathrm{GU1}\end{array}$

$-0.516300$

0.357191

0.039700

0.149086

0.064920

0.021908

0.021908

0.055394

$-0.039153$

$-0.039153$

0.680432

$-0.705580$

$-0.673557$

0.536600

$-0.581900$

\section{a $<$ TRIPOS $>$ BOND}

$\begin{array}{rrrr}1 & 1 & 2 & 1 \\ 2 & 1 & 3 & 1 \\ 3 & 3 & 4 & 1 \\ 4 & 3 & 5 & 1 \\ 5 & 3 & 14 & 1 \\ 6 & 5 & 6 & 1 \\ 7 & 5 & 7 & 1 \\ 8 & 5 & 8 & 1 \\ 9 & 8 & 9 & 1 \\ 10 & 8 & 10 & 1 \\ 11 & 8 & 11 & 1 \\ 12 & 11 & 12 & 1 \\ 13 & 11 & 13 & 1 \\ 14 & 14 & 15 & 1\end{array}$

@ $<$ TRIPOS $>$ SUBSTRUCTURE
1 GU1
1 TEMP

0

0 ROOT

\section{Coordinate file for first histidine residue in $\mathrm{Zn}^{2+}$ binding site (mol2)} a $<$ TRIPOS $>$ MOLECULE

HD1

$\begin{array}{lllll}17 & 17 & 1 & 0 & 0\end{array}$

SMALL

RESP Charge

a $<$ TRIPOS $>$ ATOM

\begin{tabular}{|c|c|c|c|c|c|}
\hline $1 \mathrm{~N}$ & -20.4740 & 7.0670 & $-14.8130 \mathrm{~N}$ & $1 \mathrm{HD} 1$ & -0.415700 \\
\hline $2 \mathrm{H}$ & -20.6500 & 6.1920 & -15.2890 & $1 \mathrm{HD} 1$ & 0.317305 \\
\hline $3 \mathrm{CA}$ & -20.8800 & 8.3100 & $-15.4600 \mathrm{CX}$ & $1 \mathrm{HD} 1$ & 0.018800 \\
\hline $\mathrm{HA}$ & -20.0040 & 8.9510 & $-15.5570 \mathrm{H1}$ & $1 \mathrm{HD} 1$ & 0.157894 \\
\hline $\mathrm{CB}$ & -21.4070 & 7.9870 & $-16.8630 \mathrm{CT}$ & $1 \mathrm{HD} 1$ & -0.222931 \\
\hline $\mathrm{HB} 2$ & -20.6360 & 7.4340 & $-17.4030 \mathrm{HC}$ & $1 \mathrm{HD} 1$ & 0.087795 \\
\hline 7 HB3 & -22.2860 & 7.3580 & $-16.8190 \mathrm{HC}$ & $1 \mathrm{HD} 1$ & 0.08779 \\
\hline $8 \mathrm{CG}$ & $-21 \cdot 7920$ & 9.1860 & $-17.6660 \mathrm{CC}$ & $1 \mathrm{HD} 1$ & 0.027037 \\
\hline ND1 & -21.1260 & 9.5500 & $-18.8200 \mathrm{NA}$ & $1 \mathrm{HD} 1$ & -0.16088 \\
\hline $10 \mathrm{HD1}$ & -20.3340 & 9.0600 & $-19.2160 \mathrm{H}$ & $1 \mathrm{HD} 1$ & 0.31331 \\
\hline $11 \mathrm{CE} 1$ & -21.6950 & 10.6280 & $-19.3310 \mathrm{CR}$ & $1 \mathrm{HD} 1$ & 0.11966 \\
\hline
\end{tabular}




$\begin{array}{rrrr}12 & \mathrm{HE} 1 & & \\ 13 & \mathrm{NE} 2 & & \\ 14 & \mathrm{CD} 2 & & \\ 15 & \mathrm{HD} 2 & & \\ 16 & \mathrm{C} & & \\ 17 & \mathrm{O} & & \\ \text { Q }<\mathrm{TRIPOS}>\mathrm{BOND} & & \\ 1 & 1 & 2 & 1 \\ 2 & 1 & 3 & 1 \\ 3 & 3 & 4 & 1 \\ 4 & 3 & 5 & 1 \\ 5 & 3 & 16 & 1 \\ 6 & 5 & 6 & 1 \\ 7 & 5 & 7 & 1 \\ 8 & 5 & 8 & 1 \\ 9 & 8 & 9 & 1 \\ 10 & 8 & 14 & 1 \\ 11 & 9 & 10 & 1 \\ 12 & 9 & 11 & 1 \\ 13 & 11 & 12 & 1 \\ 14 & 11 & 13 & 1 \\ 15 & 13 & 14 & 1 \\ 16 & 14 & 15 & 1 \\ 17 & 16 & 17 & 1\end{array}$

$\begin{array}{rrrl}-21.4030 & 11.1460 & -20.2290 & \mathrm{H} 5 \\ -22.7010 & 10.9800 & -18.5460 & \mathrm{Y} 1 \\ -22.7880 & 10.0890 & -17.5030 & \mathrm{CV} \\ -23.5370 & 10.0870 & -16.7210 & \mathrm{H} 4 \\ -21.9280 & 9.0480 & -14.6270 & \mathrm{C} \\ -21.7780 & 10.2390 & -14.3270 & \mathrm{O}\end{array}$

$1 \mathrm{HD} 1$

0.214183

1 HD1

$-0.508622$

1 HD1

0.086858

1 HD1

0.119129

$1 \mathrm{HD} 1$

0.597300

1 HD1

$-0.567900$

@ $<$ TRIPOS $>$ SUBSTRUCTURE
$1 \mathrm{HD} 1$
1 TEMP

0

0 ROOT

Coordinate file for second histidine residue in $\mathrm{Zn}^{2+}$ binding site (mol2) @ $<$ TRIPOS $>$ MOLECULE

$\mathrm{HD} 2$

$\begin{array}{lllll}17 & 17 & 1 & 0 & 0\end{array}$

SMALL

RESP Charge

a $<$ TRIPOS $>$ ATOM

\begin{tabular}{|c|c|}
\hline \multicolumn{2}{|l|}{$1 \mathrm{~N}$} \\
\hline $2 \mathrm{H}$ & \\
\hline $3 \mathrm{CA}$ & \\
\hline $4 \mathrm{HA}$ & \\
\hline $5 \mathrm{CB}$ & \\
\hline 6 HB2 & \\
\hline 7 HB3 & \\
\hline 8 CG & \\
\hline $9 \mathrm{ND} 1$ & \\
\hline HD1 & \\
\hline CE1 & \\
\hline HE1 & \\
\hline $\mathrm{NE} 2$ & \\
\hline CD2 & \\
\hline $\mathrm{HD} 2$ & \\
\hline $5 \mathrm{C}$ & \\
\hline 0 & \\
\hline $\mathrm{S}>\mathrm{BON}$ & \\
\hline 1 & 21 \\
\hline 1 & 31 \\
\hline 3 & 41 \\
\hline 3 & 5 \\
\hline 3 & 16 \\
\hline
\end{tabular}

$$
\begin{aligned}
& -21.9940 \\
& -22.2420 \\
& -23.0040 \\
& -22.6170 \\
& -24.2900 \\
& -24.6720 \\
& -25.0600 \\
& -24.2220 \\
& -23.5610 \\
& -23.1230 \\
& -23.6720 \\
& -23.3290 \\
& -24.3670 \\
& -24.7320 \\
& -25.3210 \\
& -23.3080 \\
& -23.2940
\end{aligned}
$$

12.7620

$-12.1340 \mathrm{~N}$

1 HD2

$-0.415700$

11.9150

$-12.6280 \mathrm{H}$

0.358328

13.8090

$-11.9870 \mathrm{CX}$

0.018800

14.7340

$-12.4150 \mathrm{H} 1$

0.065505

13.4080

$-12.7110 \mathrm{CT}$

$-0.091292$

12.4470

$-12.4530 \mathrm{HC}$

0.032721

14.1120

$-12.3910 \mathrm{HC}$

0.032721

13.5440

$-14.1910 \mathrm{CC}$

0.066406

14.5810

$-14.8120 \mathrm{NA}$

$-0.149862$

15.3590

$-14.3380 \mathrm{H}$

0.321043

14.4490

$-16.1200 \mathrm{CR}$

0.107703

15.1500

$-16.8640 \mathrm{H} 5$

0.101460

13.3530

$-16.3670 \mathrm{Y} 2$

$-0.332596$

12.7730

$-15.1790 \mathrm{CV}$

$-0.073789$

11.8820

$-15.0530 \mathrm{H} 4$

0.135267

14.0900

$-10.5150 \mathrm{C}$

0.597300

15.2470

$-10.07400$

$-0.567900$

\section{a $<$ TRIPOS $>$ BOND}




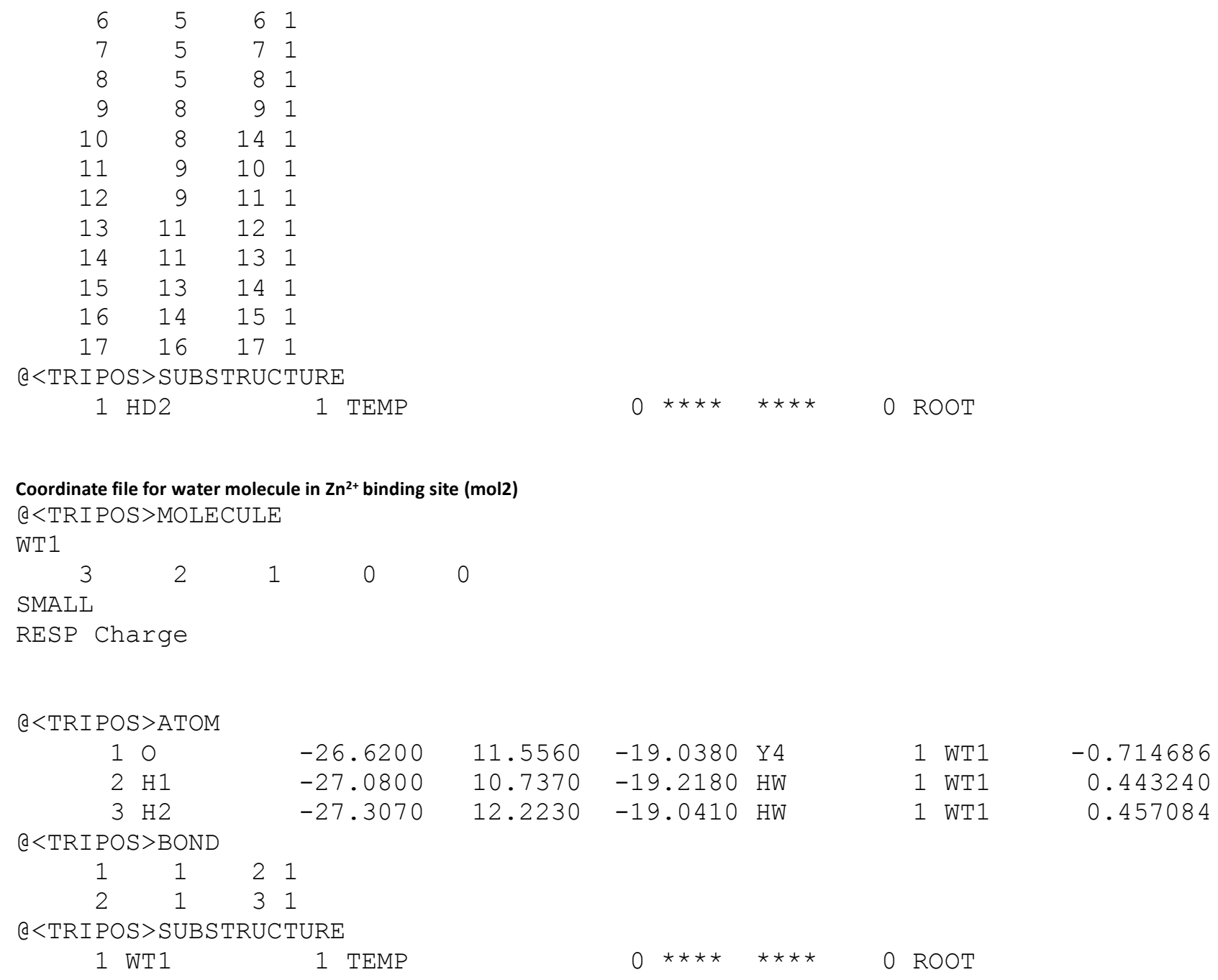

Coordinate file for $\mathrm{Zn}^{2+}$ molecule in $\mathrm{Zn}^{2+}$ binding site ( $\mathrm{mol} 2$ )

a $<$ TRIPOS $>$ MOLECULE

ZN1

SMALL

RESP Charge

a $<$ TRIPOS $>$ ATOM

$$
\begin{array}{llllllll}
1 \mathrm{ZN} & -24.7420 & 12.2140 & -18.2970 & \mathrm{M} 1 & \mathrm{ZN} 1 & 0.965715
\end{array}
$$

a $<$ TRIPOS $>$ BOND

@ $<$ TRIPOS $>$ SUBSTRUCTURE
$1 \mathrm{ZN} 1$
1 TEMP
$0 * \star \star \star * * \star * \star *$
0 ROOT

Force field parameters for $\mathrm{Zn}^{2+}$ binding site (frcmod)

REMARK GOES HERE, THIS FILE IS GENERATED BY MCPB.PY

MASS

M1 65.4

Y1 14.01

0.530

0.530

Y2 14.01

0.434

Y3 16.00

0.000

$\mathrm{Zn}$ ion

sp2 $\mathrm{N}$ in 5 memb.ring $\mathrm{w} / \mathrm{LP}$ (HIS, ADE, GUA) $\mathrm{sp} 2 \mathrm{~N}$ in 5 memb.ring $\mathrm{w} / \mathrm{LP}$ (HIS, ADE, GUA)

Y4 16.00 carboxyl and phosphate group oxygen oxygen in TIP3P water

BOND 
M1-Y4 55.6 2.0490 Created by Seminario method using MCPB.py, replaced with an harmonic restraint (hybrid bonded/restrained non bonded model)

$\begin{array}{lrl}\text { Y1-M1 } & 90.7 & 1.9829 \\ \text { Y2-M1 } & 89.3 & 1.9845 \\ \text { Y3-M1 } & 89.8 & 1.9329 \\ \text { CO-Y3 } & 656.0 & 1.2500 \\ \text { CR-Y1 } & 488.0 & 1.335 \\ \text { CR-Y2 } & 488.0 & 1.335 \\ \text { Y1-CV } & 410.0 & 1.394 \\ \text { Y2-CV } & 410.0 & 1.394 \\ \text { Y4-HW } & 553.0 & 0.9572\end{array}$

Created by Seminario method using MCPB.py Created by Seminario method using MCPB.Py Created by Seminario method using MCPB.py

JCC , 7, (1986), 230; HIS

JCC , 7, (1986), 230; HIS

$\mathrm{JCC}, 7,(1986), 230$; HIS

$\mathrm{JCC}, 7$, (1986), 230 ; HIS

Y 4-HW $553.0 \quad 0.9572$

! TIP3P water

ANGL

$\begin{array}{lll}\text { CO-Y3-M1 } & 65.54 & 114.66 \\ \text { CR-Y1-M1 } & 58.24 & 122.34 \\ \text { CR-Y2-M1 } & 31.07 & 119.08 \\ \text { M1-Y1-CV } & 59.96 & 131.01 \\ \text { M1-Y2-CV } & 31.60 & 134.25 \\ \text { M1-Y4-HW } & 41.44 & 107.64 \\ \mathrm{Y} 1-\mathrm{M} 1-\mathrm{Y} 2 & 34.77 & 118.61 \\ \mathrm{Y} 1-\mathrm{M} 1-\mathrm{Y} 3 & 27.33 & 115.72 \\ \mathrm{Y} 1-\mathrm{M} 1-\mathrm{Y} 4 & 46.78 & 100.88 \\ \mathrm{Y} 2-\mathrm{M} 1-\mathrm{Y} 3 & 61.02 & 103.02 \\ \mathrm{Y} 2-\mathrm{M} 1-\mathrm{Y} 4 & 26.72 & 115.78 \\ \mathrm{Y} 3-\mathrm{M} 1-\mathrm{Y} 4 & 54.83 & 102.13 \\ 2 \mathrm{C}-\mathrm{CO}-\mathrm{Y} 3 & 70.0 & 117.00 \\ \mathrm{CC}-\mathrm{CV}-\mathrm{Y} 1 & 70.0 & 120.00 \\ \mathrm{CC}-\mathrm{CV}-\mathrm{Y} 2 & 70.0 & 120.00 \\ \mathrm{CR}-\mathrm{Y} 1-\mathrm{CV} & 70.0 & 117.00 \\ \mathrm{CR}-\mathrm{Y} 2-\mathrm{CV} & 70.0 & 117.00 \\ \mathrm{HW}-\mathrm{Y} 4-\mathrm{HW} & 100 . & 104.52 \\ \mathrm{NA}-\mathrm{CR}-\mathrm{Y} 1 & 70.0 & 120.00 \\ \mathrm{NA}-\mathrm{CR}-\mathrm{Y} 2 & 70.0 & 120.00 \\ \mathrm{O} 2-\mathrm{CO}-\mathrm{Y} 3 & 80.0 & 126.00 \\ \mathrm{Y} 1-\mathrm{CR}-\mathrm{H} 5 & 50.0 & 120.00 \\ \mathrm{Y} 1-\mathrm{CV}-\mathrm{H} 4 & 50.0 & 120.00 \\ \mathrm{Y} 2-\mathrm{CR}-\mathrm{H} 5 & 50.0 & 120.00 \\ \mathrm{Y} 2-\mathrm{CV}-\mathrm{H} 4 & 50.0 & 120.00\end{array}$

DIHE

$\mathrm{X}-\mathrm{CR}-\mathrm{Y} 1-\mathrm{X}$

$X-C R-Y 2-X$

$\mathrm{X}-\mathrm{CV}-\mathrm{Y} 1-\mathrm{X}$

$\mathrm{X}-\mathrm{CV}-\mathrm{Y} 2-\mathrm{X}$

$2 \mathrm{C}-2 \mathrm{C}-\mathrm{CO}-\mathrm{Y} 3$

$2 \mathrm{C}-2 \mathrm{C}-\mathrm{CO}-\mathrm{Y} 3$

$2 \mathrm{C}-\mathrm{CO}-\mathrm{Y} 3-\mathrm{M} 1$

$\mathrm{CC}-\mathrm{CV}-\mathrm{Y} 1-\mathrm{M} 1$

$\mathrm{CC}-\mathrm{CV}-\mathrm{Y} 2-\mathrm{M} 1$

$\mathrm{CO}-\mathrm{Y} 3-\mathrm{M} 1-\mathrm{Y} 4$

$\mathrm{CR}-\mathrm{Y} 1-\mathrm{M} 1-\mathrm{Y} 2$

$\mathrm{CR}-\mathrm{Y} 1-\mathrm{M} 1-\mathrm{Y} 3$

$\mathrm{CR}-\mathrm{Y} 1-\mathrm{M} 1-\mathrm{Y} 4$

$\mathrm{CR}-\mathrm{Y} 2-\mathrm{M} 1-\mathrm{Y} 3$

$\mathrm{CR}-\mathrm{Y} 2-\mathrm{M} 1-\mathrm{Y} 4$

$\mathrm{M} 1-\mathrm{Y} 1-\mathrm{CR}-\mathrm{H} 5$

$\mathrm{M} 1-\mathrm{Y} 1-\mathrm{CV}-\mathrm{H} 4$

$\mathrm{M} 1-\mathrm{Y} 2-\mathrm{CR}-\mathrm{H} 5$

$\mathrm{M} 1-\mathrm{Y} 2-\mathrm{CV}-\mathrm{H} 4$

10.0
10.0
4.8
4.8
0.064
0.39
0.00
0.00
0.00
0.00
0.00
0.00
0.00
0.00
0.00
0.00
0.00
0.00
0.00

Created by Seminario method using MCPB.py Created by Seminario method using MCPB.py Created by Seminario method using MCPB.Py Created by Seminario method using MCPB.Py Created by Seminario method using MCPB.Py Created by Seminario method using MCPB.Py Created by Seminario method using MCPB.py Created by Seminario method using MCPB.py Created by Seminario method using MCPB.py Created by Seminario method using MCPB.Py Created by Seminario method using MCPB.py Created by Seminario method using MCPB.py
AA his
AA his
AA his
AA his
TIP3P water
AA his
AA his
AA his
AA his
AA his
AA his

$\begin{array}{rr}180.0 & 2.0 \\ 180.0 & 2.0 \\ 180.0 & 2.0 \\ 180.0 & 2.0 \\ 0.0 & -4.0 \\ 180.0 & 2.0 \\ 0.00 & 3.0 \\ 0.00 & 3.0 \\ 0.00 & 3.0 \\ 0.00 & 3.0 \\ 0.00 & 3.0 \\ 0.00 & 3.0 \\ 0.00 & 3.0 \\ 0.00 & 3.0 \\ 0.00 & 3.0 \\ 0.00 & 3.0 \\ 0.00 & 3.0 \\ 0.00 & 3.0 \\ 0.00 & 3.0\end{array}$

JCC $, 7,(1986), 230$

JCC $, 7,(1986), 230$

JCC $, 7,(1986), 230$

$\mathrm{JCC}, 7,(1986), 230$

Treat as zero by MCPB.py Treat as zero by MCPB.py Treat as zero by MCPB.py Treat as zero by MCPB.py Treat as zero by MCPB.py Treat as zero by MCPB.py Treat as zero by MCPB.Py Treat as zero by MCPB.py Treat as zero by MCPB.py Treat as zero by MCPB.py Treat as zero by MCPB.py Treat as zero by MCPB.Py Treat as zero by MCPB.py 


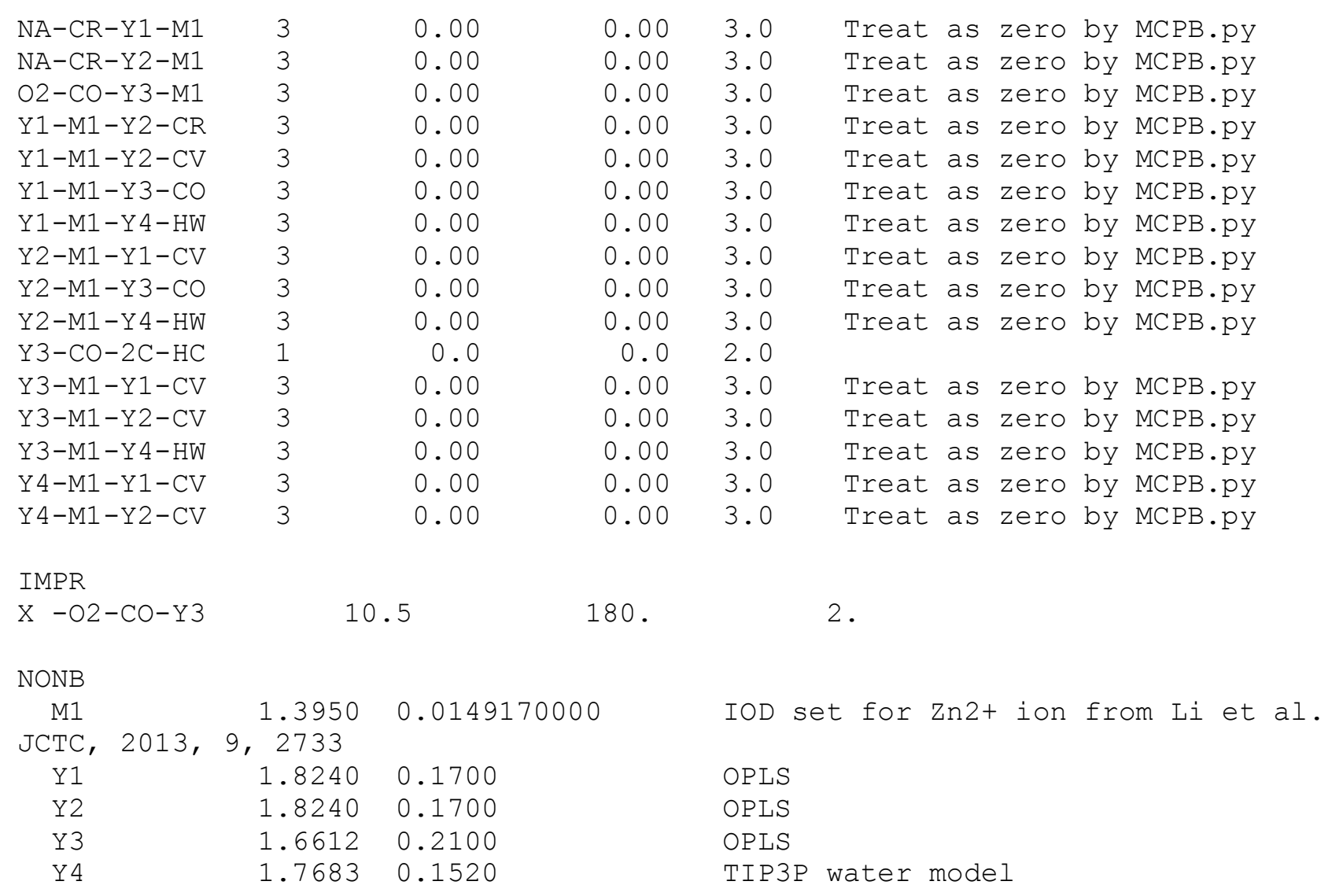

\section{References}

1. Lan, J.; Ge, J.; Yu, J.; Shan, S.; Zhou, H.; Fan, S.; Zhang, Q.; Shi, X.; Wang, Q.; Zhang, L.; Wang, X. Structure of the SARS-CoV-2 Spike Receptor-Binding Domain Bound to the ACE2 Receptor. Nature 2020, 581, 215-220.

2. Berman, H. M.; Westbrook, J.; Feng, Z.; Gilliland, G.; Bhat, T. N.; Weissig, H.; Shindyalov, I. N.; Bourne, P. E. The Protein Data Bank. Nucleic Acids Res 2000, 28, 235-42.

3. Anandakrishnan, R.; Aguilar, B.; Onufriev, A. V. H++ 3.0: Automating pK Prediction and the Preparation of Biomolecular Structures for Atomistic Molecular Modeling and Simulations. Nucleic Acids Res. 2012, 40 (Web Server issue), W537-41.

4. Pengfei, L.; Kenneth, M. M. MCPB.py: A Python Based Metal Center Parameter Builder. J. Chem. Inf. Model. 2016, 56, 599-604.

5. Case, D. A.; Ben-Shalom, I. Y.; Brozell, S. R.; Cerutti, D. S.; Cheatham, T.E. III; Cruzeiro, V. W. D.; Darden, T. A.; Duke, R. E.; Ghoreishi, D.; Giambasu, G.; Giese, T.; Gilson, M. K.; Gohlke, H.; Goetz, A .W.; Greene, D.; Harris, R.; Homeyer, N.; Huang, Y.; Izadi, S.; Kovalenko, A.; et al. AMBER 2019, University of California, San Francisco, CA, USA. 2019.

6. Gordon, M. S.; Schmidt, M. W. Advances in Electronic Structure Theory: GAMESS a Decade Later. In Theory and Applications of Computational Chemistry; Dykstra, C. E., Frenking, G., Kim, K. S., Scuseria, G. E., Eds.; Elsevier: Amsterdam, 2005; pp 1167-1189.

7. Maier, J. A.; Martinez, C.; Kasavajhala, K.; Wickstrom, L.; Hauser, K. E.; Simmerling, C. ff14SB: Improving the Accuracy of Protein Side Chain and Backbone Parameters from ff99SB. J. Chem. Theory Comput. 2015, 11, 3696-3713.

8. Kirschner, K. N.; Yongye, A. B.; Tschampel, S. M.; Gonzalez-Outeirino, J.; Daniels, C. R.; Foley, B. L.; Woods, R. J. GLYCAM06: A Generalizable Biomolecular Force Field. Carbohydrates. J. Comput. Chem. 2008, 29, 622-655. 
9. Jorgensen, W. L.; Chandrasekhar, J.; Madura, J. D.; Impey, R. W.; Klein, M. L. Comparison of Simple Potential Functions for Simulating Liquid Water. J. Chem. Phys. 1983, 79, 926-935.

10. Berendsen, H. J. C.; Postma, J. P. M.; van Gunsteren, W. F.; DiNola, A.; Haak, J. R. Molecular Dynamics with Coupling to an External Bath. J. Chem. Phys. 1984, 81, 3684-3690.

11. Toukmaji, A.; Sagui, C.; Board, J.; Darden, T. Efficient Particle-Mesh Ewald Based Approach to Fixed and Induced Dipolar Interactions. J. Chem. Phys. 2000, 113, 10913-10927.

12. Loncharich, R. J.; Brooks, B. R.; Pastor, R. W. Langevin Dynamics of Peptides: the Frictional Dependence of Isomerization Rates of N-Acetylalanyl-N'-Methylamide. Biopolymers 1992, 32, 523-535.

13. Ryckaert, J.-P.; Ciccotti, G.; Berendsen, H. J. C. Numerical Integration of the Cartesian Equations of Motion of a System with Constraints: Molecular Dynamics of n-Alkanes. J. Comput. Phys. 1977, 23, 327-341.

14. Pettersen, E. F.; Goddard, T. D.; Huang, C. C.; Couch, G. S.; Greenblatt, D. M.; Meng, E. C.; Ferrin, T. E. UCSF Chimera - A Visualization System for Exploratory Research and Analysis. J.

Comput. Chem. 2004, 25, 1605-1612.

15. Yan, Y.; Yang, M.; Ji, C. G.; Zhang, J. Z. H. Interaction Entropy for Computational Alanine Scanning. J. Chem. Inf. Model. 2017, 57, 1112-1122.

16. Sun, Z.; Yan, Y. N.; Yang, M.; Zhang, J. Z. Interaction Entropy for Protein-Protein Binding. J. Chem. Phys. 2017, 146, 124124.

17. Liu, X.; Peng, L.; Zhou, Y.; Zhang, Y.; Zhang, J. Z. H. Computational Alanine Scanning with Interaction Entropy for Protein-Ligand Binding Free Energies. J. Chem. Theory Comput. 2018, 14, 1772-1780.

18. Wang, E.; Sun, H.; Wang, J.; Wang, Z.; Liu, H.; Zhang, J. Z. H.; Hou, T. End-Point Binding Free Energy Calculation with MM/PBSA and MM/GBSA: Strategies and Applications in Drug Design. Chem. Rev. 2019, 119, 9478-9508.

19. Moreira, I. S.; Fernandes, P. A.; Ramos, M. J. Computational Alanine Scanning Mutagenesis - An Improved Methodological Approach. J. Comput. Chem. 2007, 28, 644-654.

20. Simões, I. C.; Costa, I. P.; Coimbra, J. T.; Ramos, M. J.; Fernandes, P. A. New Parameters for Higher Accuracy in the Computation of Binding Free Energy Differences upon Alanine Scanning Mutagenesis on Protein-Protein Interfaces. J. Chem. Inf. Model. 2017, 57, 60-72. 